\title{
The History of ERAS (Enhanced Recovery After Surgery) Society and its development in Latin America
}

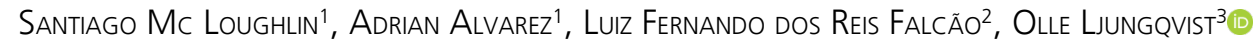

\section{A B S T R A C T}

The shortage of hospital beds and changes in the payment model have promoted an increased attention and financing of programs that focus on perioperative care efficiency in. Latin America. In this paper, Enhanced Recovery After Surgery (ERAS) programs developed by the ERAS $\otimes$ Society will be discussed. The implementation and use of ERAS ${ }^{2}$ Society Guidelines consistently demonstrated a reduction in postoperative complications, hospital stay and costs. In the current paper, the definition of ERAS programs, their core elements, and the results of their implementation and regional developments are presented with special focus on Latin America.

Keywords: ERAS. Enhanced Recovery. Implementation. Latin America.

\section{A global problem and the rise of ERAS protocols}

P opulation growth has far exceeded that of hospital infrastructure leading to a shortage of hospital beds in many countries ${ }^{1-3}$. The number and complexity of surgeries has also been steadily growing with the development of improved surgical and anesthetic techniques. Despite these improvements, the rate of major postoperative complications has been documented to occur in approximately $25 \%$ of patients undergoing surgery as inpatients ${ }^{4}$. Nearly half of the adverse events in these studies have been suggested to be preventable ${ }^{5}$. Low- and middle-income countries have the highest burden of mistreated surgical illness ${ }^{6}$.

At the same time, many countries shifted the payment model for surgical services (from "fee-forservice" towards "pay for performance"). In the "feefor-service" model, the medical team increases its income for each service provided (procedures, complementary studies and days of hospitalization). In the "pay-forperformance" model, the payment is made for a specific operation or treatment, so any complication or extension of the hospital stay generates a higher cost for the provider and thus a lower return on the fee $\mathrm{e}^{7,8}$.
All together, these demographic, infrastructure and payment model shifts have generated an increased economic interest in programs that promote the optimization of existing resources and consequently an increase in efficiency. The exponential interest in Enhanced Recovery After Surgery (ERAS) programs continues its way consolidating it as one of today's hot topic in perioperative care (Figure 1). Ultimately, beyond these factors, surgical teams continue to have the same inspiration that has always pushed them forward: To treat as many patients as possible with the best possible results.

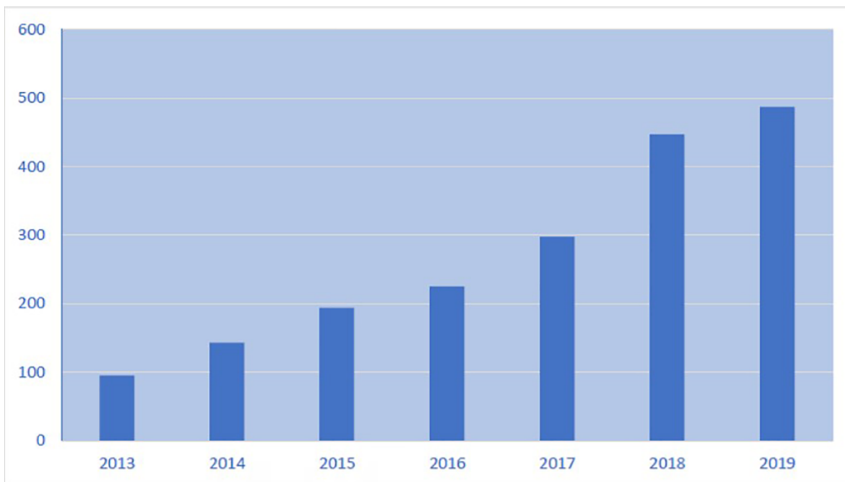

Figure 1. Number of publications indexed in PubMed per year related to Enhaced Recovery After Surgery protocols. Search criteria (ERAS + Surgery).

\footnotetext{
1 - Staff Anesthesiologist, Anesthesia Department, Hospital Italiano de Buenos Aires. Buenos Aires, Argentina. 2 - Professor of Anesthesiology, Federal University of São Paulo, Brazil. 3 - Professor of Surgery, Faculty of Medicine and Health, School of Health and Medical Sciences Department of Surgery Örebro University, Örebro, Sweden
} 
However, despite these drivers for better care, the medical community is very slow to adopt change and embrace novel treatments proven to improve outcomes for patients. For instance, fasting from midnight to the day of surgery remains standard of care in many units and countries despite no data to support its practice and changes in recommendations that data back more than 25 years ${ }^{9}$.

\section{The ERAS ${ }^{\circledR}$ Society}

Fast-track surgery was fist coined as a term for a bundle of care elements that speed up recovery time after cardiac surgery in the US ${ }^{10}$. It was further developed, conceptualized and popularized by Kehlet in colorectal surgery showing amazing improvements in recovery bringing hospital stay down from weeks to a couple of days ${ }^{11}$. The programs of the ERAS ${ }^{\circledR}$ Society represent a further development of these ideas by employing a standardized method to identify evidence based perioperative care elements that improve outcomes ${ }^{12}$ for various types of operations (www.erassociety.org). The ERAS ${ }^{\circledR}$ Society is a non for profit multi professional and multi-disciplinary medical society that had its origins in Europe at the beginning of the millennium as an initiative of several surgical groups interested in promoting a multimodal and integral care of the surgical patient. Formally, it was founded as Enhanced Recovery After Surgery for Perioperative Care (ERAS ${ }^{\circledR}$ Society) in 2010 and since then has been serving as an important platform for the expanding of ERAS programs into many specialties and surgeries and geographically throughout the world (Figure 2). The objectives of the Society are to improve outcomes for patients undergoing surgery by scientific and educational activities. A special focus is to help units worldwide to implement the ERAS ${ }^{\circledR}$ Society guidelines in a systematic way. Undoubtedly, an improved recovery leads to a decrease in hospital stay but the main objective is the quality of perioperative care and not its speed.

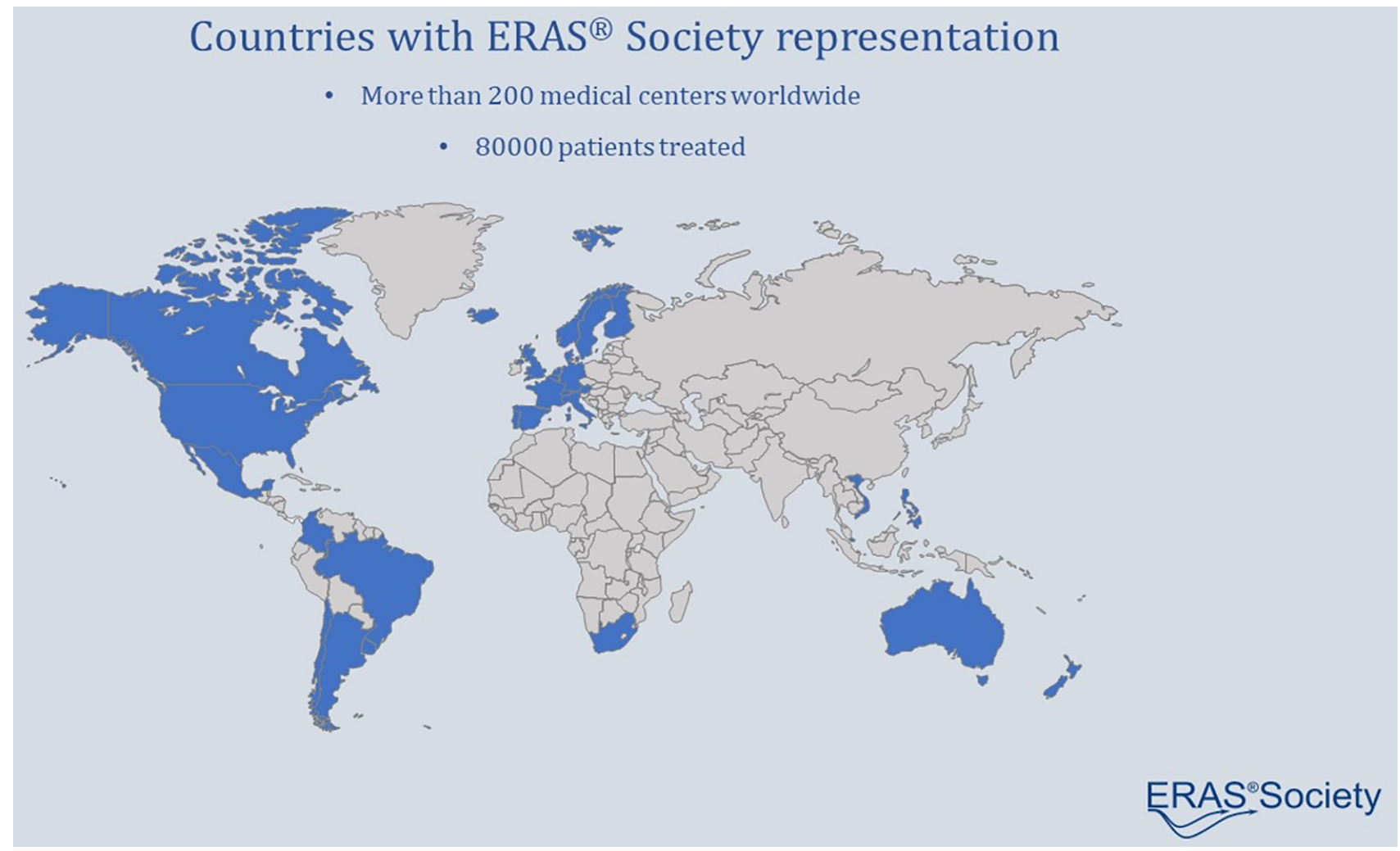

Figure 2. Active ERAS programs in the world. Countries with ongoing ERAS programs are presented with a blue background. 


\section{ERAS $^{\circledR}$ Society Implementation Programs}

ERAS ${ }^{\circledR}$ Society Implementation Programs rely on evidence-based recommendations from the Guidelines as a road map to guide clinical care. Regardless of the content of each clinical guideline (each guide is a specific for a group of surgical procedures). A distinguishing feature of the ERAS ${ }^{\circledR}$ Society is the implementation of the Guidelines using data and continuous interactive audit. The change management is based on four elements: (a) plan an intervention from the analysis of baseline data, (b) act on the plan made, (c) audit the effect and compliance to the action and finally (d) adjust care according to the new data analysis. This work cycle is part of a structured implementation program by each surgical team in their center with their patients. The fundamental tool for its implementation is the systematic registration of each patient in a unified database for all centers and its use by the unit for continuous audit of processes and outcomes.

\section{How to implement the ERAS ${ }^{\circledR}$ Society program}

All ERAS ${ }^{\circledR}$ Society implementation programs (www.erassociety.org) are run by trainers that have been through the formal ERAS® Implementation program and further training to become trainers. These trainers follow all teams of any Implementation program from start to finish and coach each unit individually. This is necessary since care varies very much from one hospital to another, from one department to another and even from one induvial doctor to another ${ }^{13,14}$. The Implementation program begins with the formation of a local multidisciplinary work team that sets up weekly meetings where they will analyze the situation and plan actions. These weekly meetings with representatives of different areas (anesthesia, surgery, nursing, nutrition, etc.) are key to addressing and controlling the different stages of surgical care entirely.
The team is constituted by a project medical leader, usually a surgeon or anesthesiologist, whose function is to assume the medical responsibility of the program before patients and authorities, sets the team's objectives and manage the necessary resources. A program coordinator, who sets the meetings, facilitates interaction between the different disciplines involved having a key role in unifying the stages (pre-surgical, intra and postsurgical) and specialties (nutritionists, kinesiologists, etc.) of the perioperative care of each patient. Finally, one team member is dedicated to the systematic collection of each patient in the interactive audit system. This is key to get the best results, since it has been reported that training without audit is not sufficient to achieve the best compliance and the best results ${ }^{15}$.

Once the team is formed, its members will carry out a training stage consisting of 4 seminars separated by 3 periods of action guided by certified instructors of the ERAS ${ }^{\circledR}$ Society. The first seminar consists of an introduction to the main elements of the guidelines and training in the use of the ERAS ${ }^{\circledR}$ Interactive Audit System (EIAS) (www.erassociety. org) that helps units control their care by keeping track of processes and outcomes related to the guidelines. The period of action that follows this first seminar is the collection of data into EIAS from the first 30 to 50 consecutive patients after the first seminar. During this period, the multidisciplinary team continues its usual practice without making any changes but collecting all the information of these patients. This group of patients (preERAS) will be considered the baseline sample. The second seminar is mainly dedicated to reviewing the results of this baseline sample of patients with the instructors. The analysis of these baseline patients shows in many cases a great discrepancy between what the surgical team believe that their common practice is and what actually happens. 
This phenomenon is not exclusive to our region, a recent study conducted in the United States showed that patients receive on average only $55 \%$ of the guidelines recommendations that are allegedly followed by the medical centers ${ }^{16}$.

Thus, it is only during the second session that the team will for the first time be able to see what is actually going on in their unit by using the audit tool to reveal not only outcomes but also processes of care that will help explain why they may have certain adverse outcomes. Based on the data collected by the team (Pre-ERAS data), a goal is defined (for example, to increase the adherence to the one or several elements of the guidelines). Now begins the second period of action. During this period, the team works to increase the adherence to the practice that they had previously set as their objective and continues to systematically record these first ERAS patients (at least consecutive 10 patients). The third seminar is dedicated to reviewing these new ten patients and determining what new objectives will be pursued. Again, a period of work of the team begins during which patient data is collected to reach approximately consecutive 50 cases, thus reaching the fourth seminar. In this last seminar the patients of the ERAS phase are reviewed and compared to the Pre-ERAS patients. The change in daily practice and outcomes that the team had set are reviewed and the global compliance to the recommendations in the guidelines is analyzed. If the overall compliance is greater than $70 \%$ or if the compliance was double as compare to the PreERAS sample, the training is considered complete. The other key objective of the implementation program is to secure a functional ERAS team that is capable of making appropriate changes based on continuous audit. This way the unit will be ready to make the next change to further improve care when new information is coming though research and updated guidelines.

\section{Building a platform for research}

As mentioned before, reliable data is indispensable for the understanding and knowledge of performance of a unit and distinctive feature of the implementation of ERAS. Without data, it is impossible to have a diagnosis of the baseline scenario or to adjust the process of care correctly. However, in many countries, including those in Latin America, information is often collected poorly by governments or large institutions. This makes it hard to run efficient health policies and may generate waste of money, time and energy. The ERAS ${ }^{\circledR}$ Society has recognized this need for useful data and developed a system that functions in two ways as a quality registry for reporting, comparisons and research as well as a day to day tool to keep track of care serving as the interactive audit.

For each group of surgeries (e.g. colorectal, liver, head and neck, etc.) the number of variables and their definition in the data base is the same worldwide. In other words, it is the same database for any hospital in the world using EIAS. This enables comparisons over time and comparisons to any other hospital of the world that participates in the Society. Information is not simply storage but rather handled in a database-management system that interacts with the users, enabling the analysis and projections of different indicators and the use of search filters for fine tuning (Figure 3).

Beyond its use to adjust our daily practice, a single global database allows an extraordinary opportunity to perform multicenter analyzes. A retrospective investigation originated in a center in Brazil can quickly become a multicenter study by using the records of other surgical teams in the world that agree to join their initiative. Currently, the EIAS harbors of more than eighty thousand patients from approximately 200 centers providing an exceptional tool to enhance research in the Latin-Americans surgical teams. 


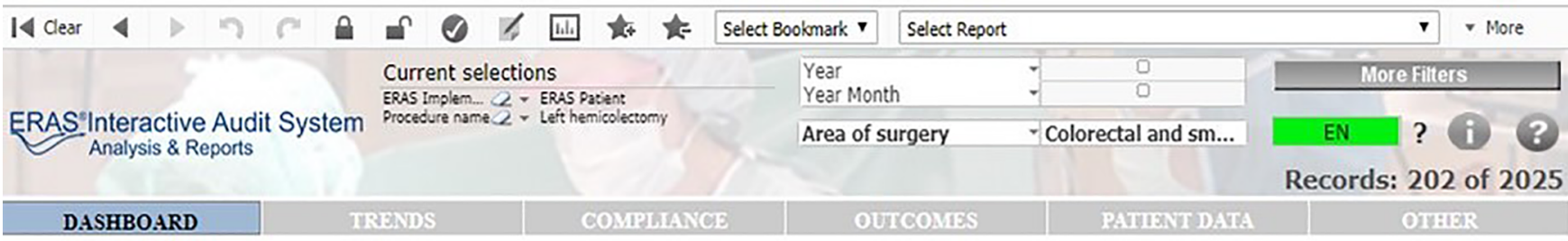

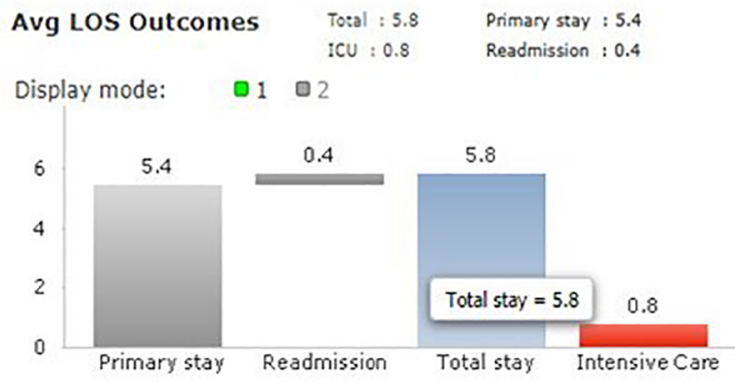

Complications (Primary and Flw-up)

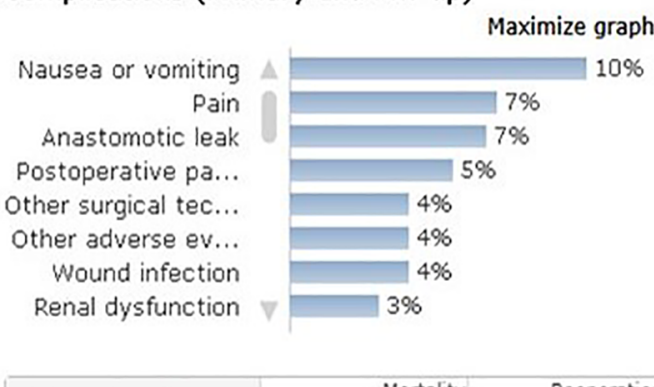

Mortality $2.0 \%$ Reoperation
$10.9 \%$

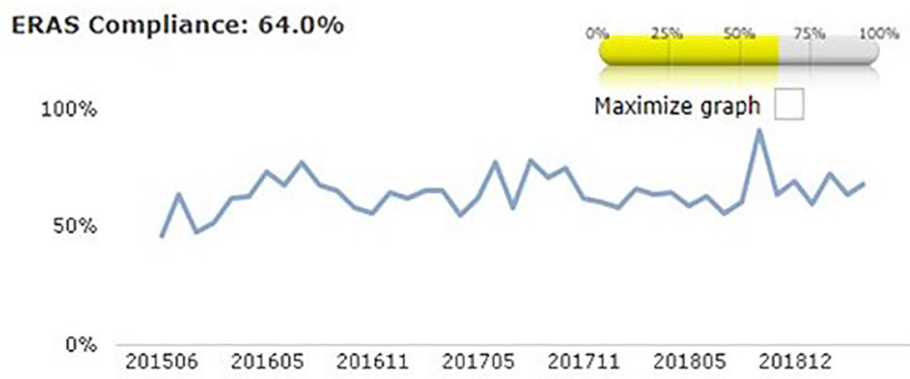

Number of Operations

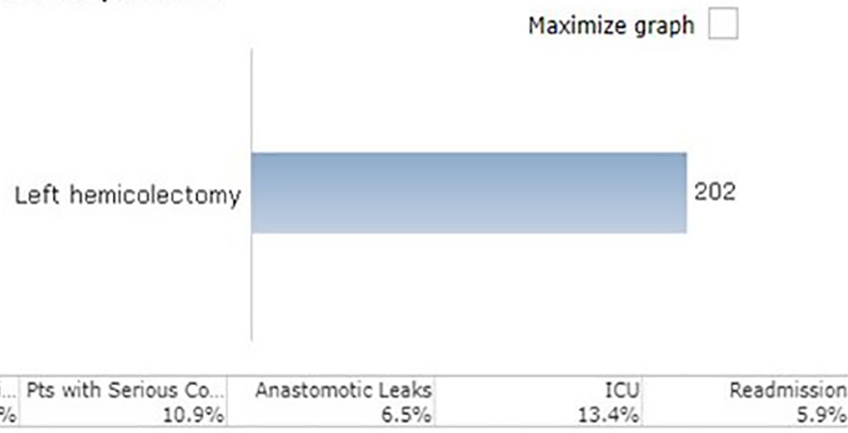

Figure 3. Eras Interactive Auditing System initial dashboard.

When performing prospective trials, currently the necessary components of a trial are usually brought together for a discrete period of time and disbanded once the trial is completed in a "one-off" manner. Significant time, energy, and money are spent on bringing the disparate resources for each trial limiting the capacity of surgical teams in our region to lead this kind of research. The constant standardized record on the EIAS platform enables to streamline the clinical trials infrastructure on resources already in place and capturing all data that have been shown to impact outcomes. This efficiency has the potential to greatly reduce the costs of research opening a great opportunity in the field of multicenter prospective trials on our region.

\section{ERAS clinical outcomes and ERAS ${ }^{\circledR}$ Society in Latin America}

ERAS programs began their development in colorectal surgery and the bulk of the experience is based on this group of surgeries. Several publications have consistently demonstrated a reduction in hospital stay in patients undergoing colorectal surgery under ERAS programs ${ }^{17,18}$. These results have been observed in both open and laparoscopic surgery ${ }^{19}$, over total length of stay or ICU stay and even in patients with a high morbidity and mortality burden ${ }^{20}$. Moreover, there are reports showing that the greater the percentage adherence to the recommendations of the ERAS ${ }^{\circledR}$ Society guidelines, the greater the reduction in hospital stay. 
Similarly, greater adherence to ERAS guidelines has shown a positive impact on postoperative complications and care costs. Recent data have also associated improved compliance with both short term survival ${ }^{15}$ and longer cancer survival after colorectal cancer surgery ${ }^{21}$.

These positive results in the field of colorectal surgery have encouraged the application of ERAS $^{\circledR}$ programs on other areas of general surgery and even other surgical specialties. Beneficial results have been observed on hospital stay and postoperative complications in thoracic surgery, gastrectomy, liver resections. Similarly, the results in orthopedic surgery, urology and gynecology are encouraging and show benefits attributable to the application of programs designed specifically for each specialty or type of surgery.

In Latin America, based on the ERAS guidelines, Dr Aguilar from Brazil first developed a multimodal approach in the wards of its Surgical Clinic Hospital ("Hospital Universitário Júlio Muller - HUJM") dedicated to accelerating the recovery of patients undergoing abdominal operations approximately ten years ago. This project, named ACERTO, successfully promotes and organizes annual seminars and courses for the diffusion of the enhanced recovery after surgery principles that is well known in Latin America and has helped spreads the ideas of ERAS on the continent. While the work of ACERTO has been supported by the ERAS $^{\circledR}$ Society, the starting point of the Society's activities in Latin America in its current form was the implementation program held at St Marks hospital in London UK for the "Hospital Italiano de Buenos Aires" in Argentina. This resulted in the first ERAS ${ }^{\circledR}$ Society appointed Center of Excellence of the region in 2014, ready to train others according to the ERAS ${ }^{\circledR}$ Implementation Program.
In addition, team members from this Center of Excellence were trained to spread the word of ERAS in the region through national symposia, congresses, and other academic meetings. Thanks to all these efforts, ERAS programs continue to expand steadily in the region and several teams followed Argentina in the implementation of ERAS programs. In the year 2015, a team from Colombia ("Clinica Reina Sofia Org Sanitas") and one from Mexico ("Hospital Civil de Guadalajara") started the path of an ERAS implementation program. In the following year, 2 hospitals from Brazil ("Hospital Vila Santa Catarina - OSS HIAE" at São Paulo and "Santa Casa de Misericordia de Porto Alegre") started and successfully completed the ERAS implementation program. In 2016, 2 institutions from Uruguay ("Hospital de Carmelo" and "Médica Uruguaya Corporación de Asistencia Médica" of Montevideo) joined our efforts for an enhanced perioperative care in the region. During 2018 and 2019, one center from Chile ("Clinica Alemana de Santiago") and the "Instituto Nacional de Cancerologia" of Mexico also joined the ERAS ${ }^{\circledR}$ LatAm initiative. In 2019, São Paulo State Society of Anesthesiology and Brazilian Society of Anesthesiology signed a MOU with ERAS ${ }^{\circledR}$ Society and ERAS ${ }^{\circledR}$ LatAm, and by the end of 2020, 8 new hospitals will be trained in Brazil on ERAS ${ }^{\circledR}$ programs for bariatric surgery ("Rede de Hospitais São Camilo de SP" and "Hospital Evangélico de Londrina") and colorectal surgery ("Instituto do Câncer de São Paulo", "Hospital 9 de Julho", "Hospital Brasília", "Hospital Santa Paula", "Hospital São Lucas" and "Complexo Hospitalar de Niterói").

This strong work has already shown beneficial results similar to the ones reported in Europe and North America. A preliminary overview of previously unpublished data shows that so far, almost 1700 patients have been included in our ERAS ${ }^{\circledR}$ LatAm register on the EIAS. 
Regional median compliance to ERAS guidelines increased from around $35-40 \%$ in 2014 up to $65-70 \%$ in 2018. At the same time, on average, ERAS implementation was associated with an approximately 2 day decrease in hospital stay, and reductions in severe complications and intensive care unit (ICU) stay in participating hospitals. These early and preliminary results suggests that ERAS works very well in this part of the world too and its implementation could come to play an important role. Hypothetically, the extension of these results to the approximately 25 million people per year undergoing major surgery in Latin America could result in an annual 50 million days reduction in the length of hospital stay.

\section{Conclusions and future perspectives}

In this paper we reviewed the definition of ERAS programs, how the ERAS Society works with implementation of the ERAS Guidelines using continuous and the first preliminary results on clinical outcomes in Latin America from these activities. Encouraged by positive regional results and feedback, our objectives for the near future are to continue the expansion of ERAS in Latin America, to strengthen regional multi-centric research helped by the use of the unified data base and finally to develop sustainability projects and research to ensure a continuous improvement of surgical care.

\section{Disclosures}

SML: Nothing to declare

AA: Founder and president ERAS Society Latin American Chapter - Vice president of implementation ERAS Society - MSD Global Anesthesia and Surgery Expert Advisory Board

LFDR: Key opinion leader and board advisor of Merck-Sharp \& Dohme and Pfizer.

Leader of ERAS ${ }^{\circledR}$ Implementation in Brazil
OL: Founder and chairman of ERAS Society, founder and shareholder Encare $A B$, speaker and travel honoraria from Fresenius Kabi, Nutricia, Encare. Advise Nutricia.

\section{REFERENCES}

1. NHS. Admissions and Bed management in NHS acute [Internet]. 2000. Available from: https:// www.nao.org.uk/report/inpatient-admissions-andbed-management-in-nhs-acute-hospitals.

2. (OECD) O for EC and D. OECD (2019), Hospital beds (indicator). (Accessed on 30 Oct). 2018.

3. Meara JG, Leather AJ, Hagander L, Alkire BC, Alonso N, Ameh EA et. al. Global Surgery 2030: evidence and solutions for achieving health, welfare, and economic development. Lancet. 386(9993):569-624.

4. Weiser TG, Haynes AB, Molina G, Lipsitz SR, Esquivel MM, Uribe-leitz T, et al. Surgical Services : Access and Coverage Estimate of the global volume of surgery in 2012 : an assessment supporting improved health outcomes. Lancet. 2012;385(Suppl2):94305.

5. Suliburk JW, Buck QM, Pirko CJ, Massarweh $\mathrm{NN}$, Barshes NR, Singh $\mathrm{H}$, et al. Analysis of Human Performance Deficiencies Associated With Surgical Adverse Events. JAMA Netw open. 2019;2(7):e198067.

6. Alkire BC, Raykar NP, Shrime MG, Weiser TG, Bickler SW, Rose JA, et al. Global access to surgical care: A modelling study. Lancet Glob Heal. 2015;3(6):e316-23.

7. Davis K. Paying for Care Episodes and Care Coordination. N Engl J Med. 2017;356(11):1166-8.

8. Hale D. Pay for Performance - Are You Prepared? Female Pelvic Med Reconstr Surg. 2019;22(3):2015-7.

9. Merchant RN, Chima N, Ljungqvist O, Kok JNJ. Preoperative Fasting Practices Across Three Anesthesia Societies: Survey of Practitioners. JMIR Perioper Med. 2020;3(1):e15905.

10. Engelman RM, Rousou JA, Flack JE, Deaton DW, Humphrey $\mathrm{CB}$, Ellison $\mathrm{LH}$, et al. Fast-track recovery of the coronary bypass patient. Ann Thorac Surg. 1994;58(6):1742-6. 
11. Kehlet $H$, Mogensen $T$. Hospital stay of 2 days after open sigmoidectomy with a multimodal rehabilitation programme. Br J Surg. 1999;86(2):227-30.

12. Brindle M, Nelson G, Lobo DN, Ljungqvist O, Gustafsson UO. Recommendations from the ERAS ${ }^{\circ}$ Society for standards for the development of enhanced recovery after surgery guidelines. BJS open. 2020;4(1):157-63.

13. Brown EG, Bateni SB, Burgess D, Li CS, Bold RJ. Interhospital Variability in Quality Outcomes of Pancreatic Surgery. J Surg Res [Internet]. 2019;235:453-8. Available from: https://doi. org/10.1016/j.jss.2018.10.035

14. Schootman $M$, Lian $M$, Pruitt $S L$, Deshpande $A D$, Hendren $S$, Mutch $M$, et al. Hospital and geographic variability in thirty-day all-cause mortality following colorectal cancer surgery. Health Serv Res. 2014;49(4):1145-64.

15. Ripollés-Melchor J, Ramírez-Rodríguez JM, CasansFrancés R, Aldecoa C, Abad-Motos A, Logroño-Egea M, et al. Association between Use of Enhanced Recovery after Surgery Protocol and Postoperative Complications in Colorectal Surgery: The Postoperative Outcomes Within Enhanced Recovery after Surgery Protocol (POWER) Study. JAMA Surg. 2019;154(8):725-36.

16. McGlynn EA, Asch SM, Adams J, Keesey J, Hicks J, DeCristofaro A KE. The Quality of Health Care Delivered to Adults in the United States. N Engl J Med. 2003;348(26):2635-45.

17. Gustafsson UO, Hausel J, Thorell A, Ljungqvist O, Soop M, Nygren J; Enhanced Recovery After Surgery Study Group. Adherence to the Enhanced Recovery
After Surgery protocol and outcomes after colorectal cancer surgery. Arch Surg. 2011;146(5):571-7.

18. Gillissen F, Hoff C, Maessen JM, Winkens B, Teeuwen $\mathrm{JH}$, von Meyenfeldt MF et. al. Structured Synchronous Implementation of an Enhanced Recovery Program in Elective Colonic Surgery in 33 Hospitals in The Netherlands. World J Surg. 2013;37(5):1082-93.

19. Levy BF, Scott MJ, Fawcett WJ, Rockall TA. 23-Hourstay laparoscopic colectomy. Dis Colon Rectum. 2009;52(7):1239-43.

20. Delaney CP, Fazio VW, Senagore AJ R, B, Halverson AL RF. Fast track postoperative management protocol for patients with high co-morbidity undergoing complex abdominal and pelvic colorectal surgery. $\mathrm{Br}$ J Surg. 2001;88(11):1533-8.

21. Gustafsson UO, Oppelstrup H, Thorell A, Nygren $J$, Ljungqvist $O$. Adherence to the ERAS protocol is Associated with 5-Year Survival After Colorectal Cancer Surgery: A Retrospective Cohort Study. World J Surg. 2016;40(7):1741-7.

Received in: 04/03/2020

Accepted for publication: 04/03/2020

Conflict of interest: None

Financing source: None

\section{Mailing address:}

Santiago Mc Loughlin

E-mail: santimcl@gmail.com

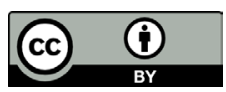

